\title{
Construction of High-accuracy Ensemble of Classifiers
}

\author{
Hedieh Sajedi \\ Department of Computer Science, School of Mathematics, Statistics and Computer Science, College of Science, \\ University of Tehran, Tehran, Iran \\ E-mail: hhsajedi@ut.ac.ir \\ Elham Masoumi \\ Department of Computer Engineering, Qazvin Branch, Islamic Azad University, Qazvin, Iran
}

\begin{abstract}
There have been several methods developed to construct ensembles. Some of these methods, such as Bagging and Boosting are meta-learners, i.e. they can be applied to any base classifier. The combination of methods should be selected in order that classifiers cover each other weaknesses. In ensemble, the output of several classifiers is used only when they disagree on some inputs. The degree of disagreement is called diversity of the ensemble. Another factor that plays a significant role in performing an ensemble is accuracy of the basic classifiers. It can be said that all the procedures of constructing ensembles seek to achieve a balance between these two parameters, and successful methods can reach a better balance. The diversity of the members of an ensemble is known as an important factor in determining its generalization error. In this paper, we present a new approach for generating ensembles. The proposed approach uses Bagging and Boosting as the generators of base classifiers. Subsequently, the classifiers are partitioned by means of a clustering algorithm. We introduce a selection phase for construction the final ensemble and three different selection methods are proposed for applying in this phase. In the first proposed selection method, a classifier is selected randomly from each cluster. The second method selects the most accurate classifier from each cluster and the third one selects the nearest classifier to the center of each cluster to construct the final ensemble. The results of the experiments on well-known datasets demonstrate the strength of our proposed approach, especially applying the selection of the most accurate classifiers from clusters and employing Bagging generator.
\end{abstract}

Index Terms - Classifier Ensembles, Bagging, Boosting, Decision Tree

\section{Introduction}

Classifier systems based on the combination of outputs of a set of different classifiers have been proposed in the field of pattern recognition as a method of developing high performance classification systems [1]. In ensemble method for classification, many classifiers are combined to make a final prediction [2]. Ensemble methods show better performances than a single classifier in general [3]. The final decision is usually made by voting after combining the predictions from set of classifiers.

So far different ensemble learning algorithms have been proposed like Adaboost, Bagging and Arcing. Adaboost and Arc-x(h) are two ensemble algorithms that belong to Arcing family of algorithms. For $h=4$, Arc$\mathrm{x}(\mathrm{h})$ performs equally well as Adaboost. They use different weight updating rules and combine classifiers using different voting schemes [4]. By far, the most common implementation of Boosting is AdaBoost, although some newer algorithms are reported to achieve better results.

An ensemble has a vital need for diversity, that is if an ensemble of classifiers is to be good as a plural classification, they should be diverse as enough as to cover each other's' errors. Consequently, while constructing an ensemble, we need a mechanism to guarantee the diversity of the ensemble. Sometimes this mechanism is in a way that a basic classifier is selected or removed from ensemble to maintain the diversity of the ensemble. In ensemble-based learning, it is possible that the data is fractioned into smaller parts and afterward a simple and quick model is created for each part. In this regard, final ensemble model is performed in a way without significant time overhead. To learn any problem, many classifiers have been introduced so far [5]. Each of the proposed classifiers has strengths and weaknesses that make it suitable for specific problems. Nevertheless ensemble based learning is a powerful approach to provide an optimal classification system for each problem. The most challenging issue in constructing an ensemble based classification system is how constructing a proper ensemble of base classifiers [6].

In researches discussed in [7, 8], fuzzy k-means algorithm has been used for clustering the classifiers. The partitioning is done considering the outputs of 
classifiers on train dataset as feature space. From each partition, one classifier is selected to participate in final ensemble. Furthermore, in these approaches, each classifier is examined over the whole train dataset and its accuracy is calculated based on the results.

Selecting an appropriate classifier from each cluster is not investigated in the previous researches. This issue can highly effect on the accuracy of classification tasks.

In this paper, we introduce a new approach for constructing ensembles. It firstly makes base classifiers. Afterward k-means clustering algorithm is employed to partition classifiers. Then some classifiers are selected from clusters to construct the final ensemble. This approach utilizes Bagging and Boosting algorithms as producers of basic classifiers. We proposed three selection methods to select a classifier from each cluster. One of the aims of this study is to investigate whether it is possible not to select any classifier from some specific clusters and achieve a superior ensemble. To justify this situation, it has to be mentioned that in clustering of a typical dataset, some of clusters are created for a null data or some noisy data. In this paper, we investigate the effect of removing these clusters and also the effect of sampling rate of training data on the efficiency of classifiers. In our proposed approach, in order to reach the diversity required among primary classifiers, every one of them was trained with random sampled data. The volume of sampled training data is $b$ percent of total training data. Generally, in Bagging method, the value of parameter $b$ is always 100 , but in our proposed method, to produce basic classifiers, the parameter value is set to a number between 30 and 100 . Our experiments illustrated that selecting the most accurate classifier among each cluster, is led to the ensemble with highest accuracy. The most proper values for the number of clusters and the sampling rate were determined by different experiments. Furthermore, the experimental results illustrate the effectiveness of our proposed approach.

The organization of this paper is as the follows: In the second section, related works are discussed. Proposed approach is explained in Section 3 and experimental results are described in the fourth section. Section 5 includes conclusion.

\section{Related Works}

Ensemble learning is a powerful approach to provide any problem with an almost optimal classification system. Generally it is true that combining different classifiers leads to increase in classification efficiency $[9,10]$. This shows the importance of diversity in an ensemble's success. Diversity among basic classifiers signifies their independence. This means that failure in classification of basic classifiers will not take place simultaneously on one template. Kuncheva in [11] has shown that, theoretically and practically, an ensemble of some basic classifiers always increases classifications' accuracy if basic classifiers are independent. It has also been shown that ensemble philosophy can be also applied to Bayesian networks [12]. Kuncheva has proposed some methods to measure an ensemble's diversity and then has suggested a model to build a diverse ensemble based on a search method [13].

Application of clustering classifiers has been discussed in $[14,15]$. In previous works, classifiers in the ensemble have performed their learning process randomly and each one is constructed based on the entire training set. It has been proved that combining classifiers in the ensemble are more efficient than individual ones in average [17]. Since classifiers with different features or different methodology can complement each other and cover each other's disadvantages, combining methods often lead to improve classification efficiency [6]. Kuncheva in [18] demonstrates by kondorset theorem that usually a combination of classifiers, performs better than a single one, i.e. if different classifiers are used in combination, their total error may notably decrease. In the neural network field, several methods for the creation of ensembles of neural networks have been investigated [19]. Such methods basically lie on varying the parameters related to the design and training of neural networks.

Several techniques have been proposed to create diversity. For example different subsets of training samples are created and after that independent classifiers are created upon them. Bagging and Boosting methods are the most famous methods for creating ensembles [20, 21]. In these methods, rather than using all the samples, their subsets are used to train basic classifiers. The term Bagging is first used by [5] abbreviating for bootstrap aggregating. In Bagging idea, the ensemble is made of classifiers built on bootstrap copies of the train set. Using different train sets, the required diversity for ensemble is obtained. In order to promote model diversity, Bagging trains each model in the ensemble using a randomly-drawn subset of the training set [20]. Fig. 1 illustrates the training phase of Bagging method in general. Boosting involves incrementally building an ensemble by training each new classifier to emphasis the training instance that previous classifiers misclassified $[21,22]$.

In another level, features are used to produce diversity among classifiers, in a way that each classifier uses a subset of features $[18,9,23]$. In a different viewpoint, different kinds of classifiers are used in combination to make diversity [11]. In the mentioned methods, if we create many classifiers, there is a possibility that some of them always give the similar output. Therefore, after creating some classifiers with a given method, a selection mechanism should be applied to select appropriate classifiers and our proposed approach offers some solution to this issue. 


\section{Proposed Approach}

The main idea of our proposed approach is combining classifiers based on the most diverse subsets of basic classifiers. In the first phase, some basic classifiers are produced based on two famous mechanisms of creating ensembles, Bagging and Boosting. Then produced classifiers are partitioned by a clustering algorithm according to their outputs on the entire set of training data. Fig. 2 shows the block diagram of the proposed approach, which uses k-means clustering algorithm for portioning base classifiers.
After constructing a large number of basic classifiers, proposed approach partitions them with the help of kmeans clustering algorithm. Then the selection phase is triggered. This section involves three different selection methods. In the first method, a classifier is selected randomly from each cluster. In the second method, the most accurate classifier in each cluster is selected and in the third one, the nearest classifier to the center of each cluster is used for constructing the final ensemble.

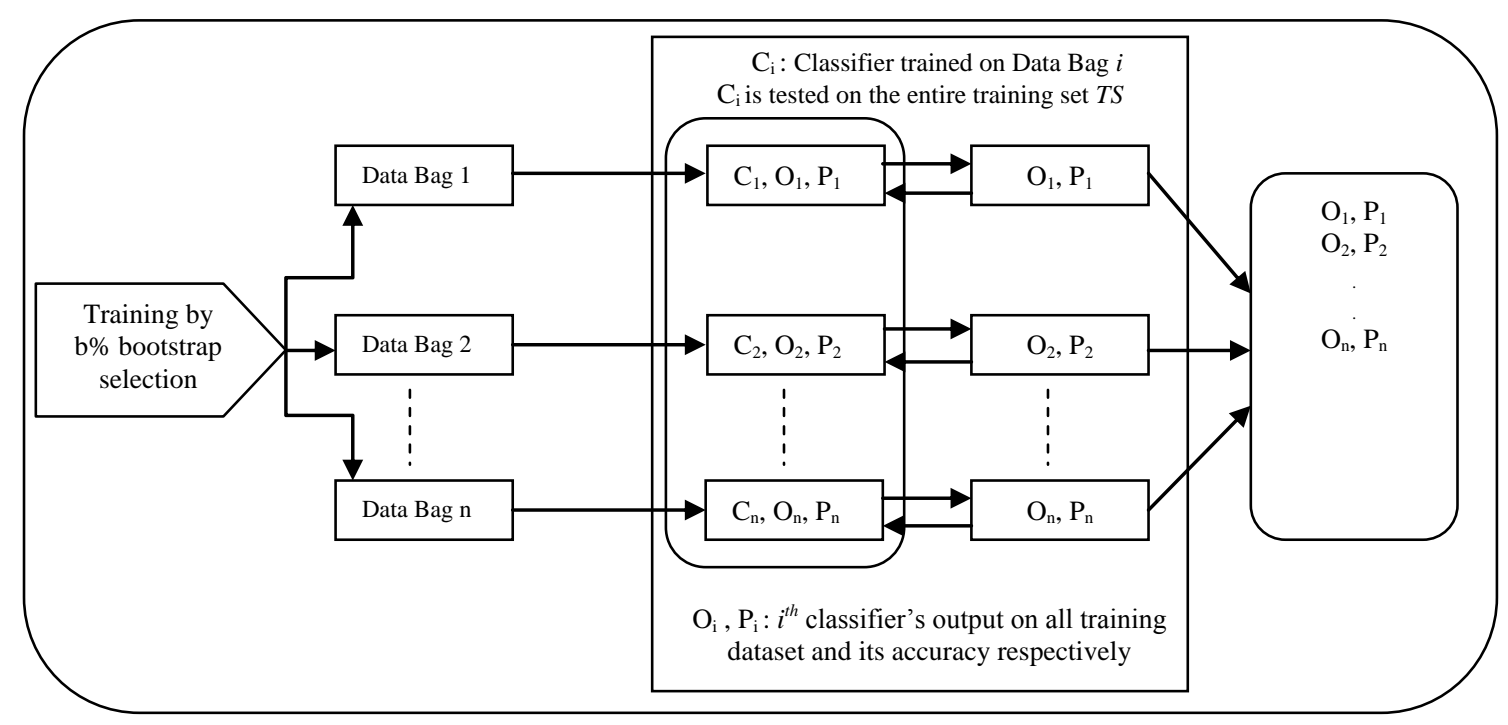

Fig. 1: Training phase of Bagging method

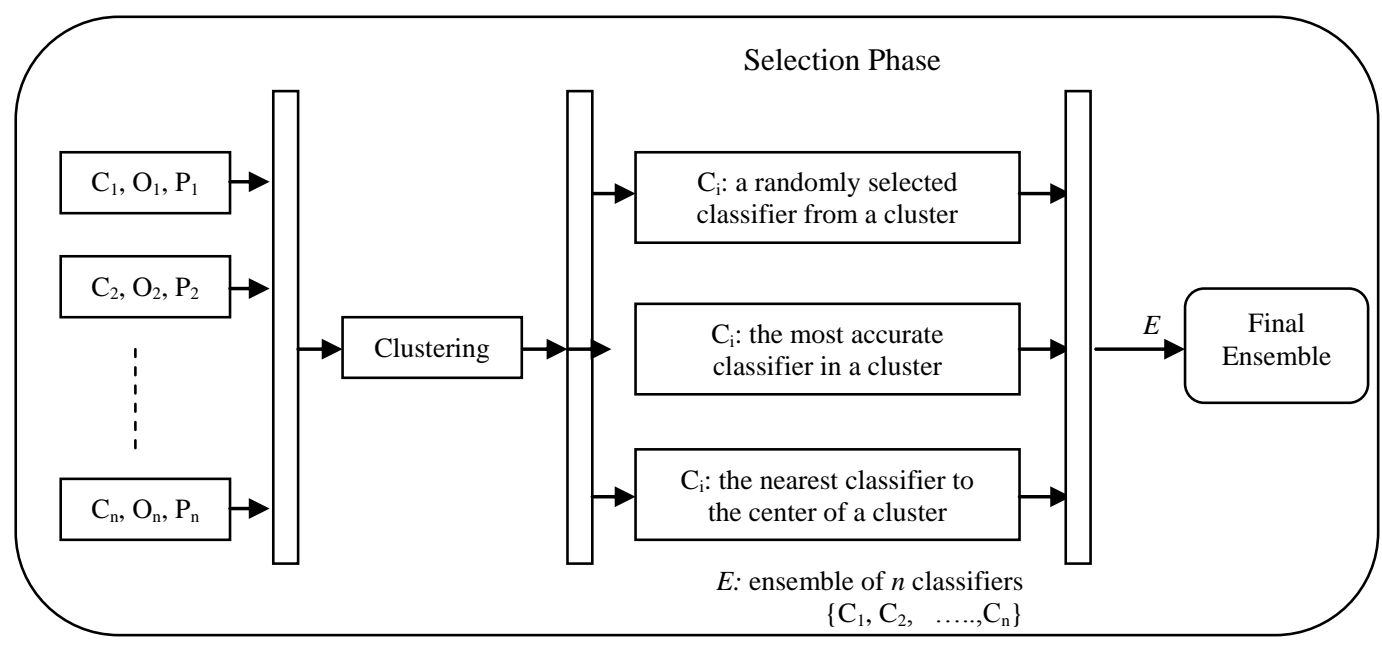

Fig. 2: Block diagram of the proposed approach

Since each cluster is created based on the outputs of classifiers, it is so likely that by selecting one classifier from each cluster and employing it to construct an ensemble, the formed ensemble is a diverse one and has better performance than ensembles created by original Bagging and Boosting approaches.
The pseudo code of the proposed approach in training part is shown in Fig. 3. In this figure, Bagging technique is used to create basic classifiers.

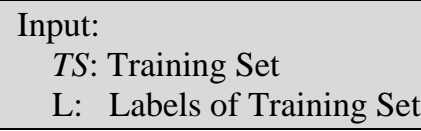




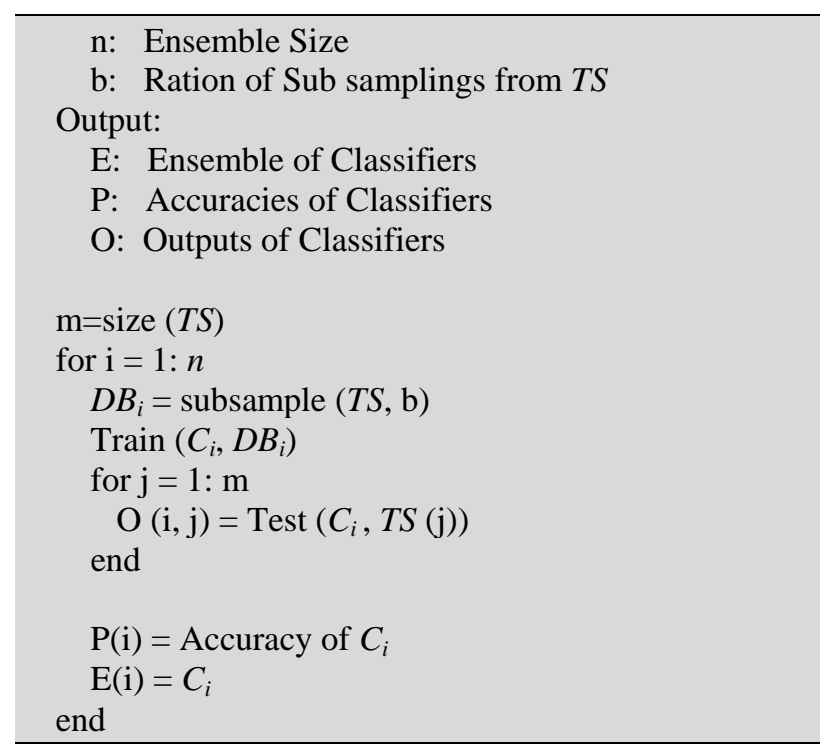

Fig. 3: The pseudo code of proposed approach, training phase

$n$ subsets of training set are provided by sampling without displacement. $i^{\text {th }}$ data subset, which is $b \%$ of training set and obtained by sampling, is called $i^{\text {th }}$ data bag and is shown as $D B_{i}$. This is obvious that the number of samples in $D B_{i}$ is $m * b / 100$ where $m$ is the number of all samples in dataset $T S$. Then a basic classifier is trained on each $D B_{i}$ and classifier trained on $D B_{i}$ is called $C_{i}$. Afterward, $C_{i}$ is tested on the entire training set $T S$ and its accuracy is calculated. The output of $i_{\text {th }}$ classifier on $j^{\text {th }}$ data from $T S$ is a vector shown by $O_{i j} . O^{k}{ }_{i j}$ is the degree of $i^{\text {th }}$ classifier's certainty that $j^{\text {th }}$ data belongs to $k^{\text {th }}$ class. $i^{\text {th }}$ classifier's output on all training datasets is shown by $O_{i}$ and its accuracy is shown by $P_{i}$. One of the differences between approaches of producing basic classifiers in proposed method and Bagging method lies in sampling rates. In Bagging method, the value of sampling rate, $b$, is always 100 but in our proposed approach, to produce basic classifiers the parameter value is set to a number between 30 and 100.

In the following, we will describe the methods proposed to employ in selection phase of the presented approach.

\subsection{Random selection Mechanism}

The pseudo code of the proposed approach by selection a classifier randomly from each cluster is shown in Fig. 4. In this method, the clusters that have less than two classifiers are deleted. Then from each cluster, a classifier is chosen randomly. $S C$ is ensemble classifier and $S P$ is accuracy of the classifier. In the framework of the proposed method, a set of samples $D C$ is created at first in which, each sample is a classifier. The $\mathrm{i}^{\text {th }}$ sample of $D C$ is the $i^{\text {th }}$ classifier. Assume that our data has $f$ features. $P^{\text {th }}$ feature of $i^{\text {th }}$ sample is shown as $X_{i p}$, that is obtained from (1):

$$
X_{i p}=o_{i j}^{k}
$$

where $j$ and $k$ are calculated by (2) and (3), in which $c$ is the number of classes.

$$
\begin{aligned}
& j=\lceil p / c\rceil \\
& k=p-j^{*} c
\end{aligned}
$$

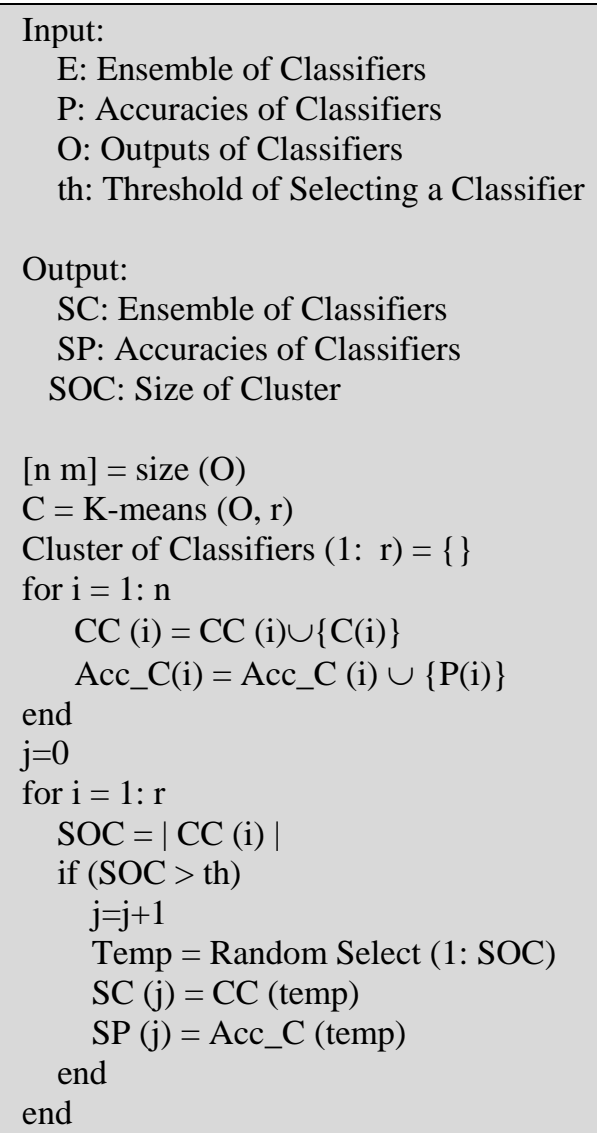

Fig. 4: The pseudo code of the proposed approach using random selection mechanism

Features of $D C$ dataset are viewpoints of different basic classifiers on main dataset's data. So we have a new dataset with $n$ data (each of which is a classifier) and $f$ features $\left(F=m^{*} c\right)$. Parameter $n$, which is a predefined parameter by user, is the number of classifiers produced by Bagging or Boosting approaches. After creating dataset $D C$, we divide it to many partitions by a clustering algorithm. The number of partitions is shown by $r$.

Classifiers in a partition have similar outputs. This means that they have low diversity. So it is better to select one classifier from each cluster. In order to avoid null classifications, clusters with fewer members than a threshold th are omitted. Assume that $E$ is an ensemble of $n$ classifiers $\left\{C_{l}, C_{2} \ldots C_{n}\right\}$ and there is $c$ classes in the problem. Assume that by applying the ensemble on data $O_{j}$, a matrix $D^{j}$ similar to (4) is resulted: 


$$
D^{j}=\left[\begin{array}{cccc}
O_{1 j}^{1} & O_{2 j}^{1} & \cdot & O_{n j}^{1} \\
\cdot & \cdot & \cdot & \cdot \\
O_{1 j}^{c-1} & O_{2 j}^{c-1} & \cdot & O_{n j}^{c-1} \\
O_{1 j}^{c} & O_{2 j}^{c} & \cdot & O_{n j}^{c}
\end{array}\right]
$$

Now ensemble decides whether data $O_{j}$ belongs to class $q$ according to (5):

$$
q=\arg \max _{i=1}^{c}\left|\sum_{k=1}^{n} w_{k} * D_{i}^{j} k\right|
$$

in which $w_{k}$ is the weight of $k^{\text {th }}$ classifier, and in optimal situation computed by (6) ${ }^{[11]}$ :

$$
w_{k}=\log \frac{p^{k}}{1-p^{k}}
$$

where $p_{k}$ is the weight of $k^{\text {th }}$ classifier which effects on training set $T S$. Notice that in equal terms we use random function in (5). Assume vector $L^{j}$ for data $O_{j}, L^{j}{ }_{O}$ is one if $O_{j}$ belongs to class $q$ and is zero if not. Now the accuracy of classification $C_{k}$ on $T S$ is calculated from (7).

$$
p_{k}=\frac{\sum_{j=1}^{m} \sum_{i=1}^{c}\left|L_{i}^{j}-O_{k j}^{i}\right|}{c \times m}
$$

\subsection{Accurate Selection Mechanism}

In this selection method, the algorithm selects the most accurate classifier from each cluster. Pseudo code of the proposed selection method is shown in Fig. 5.

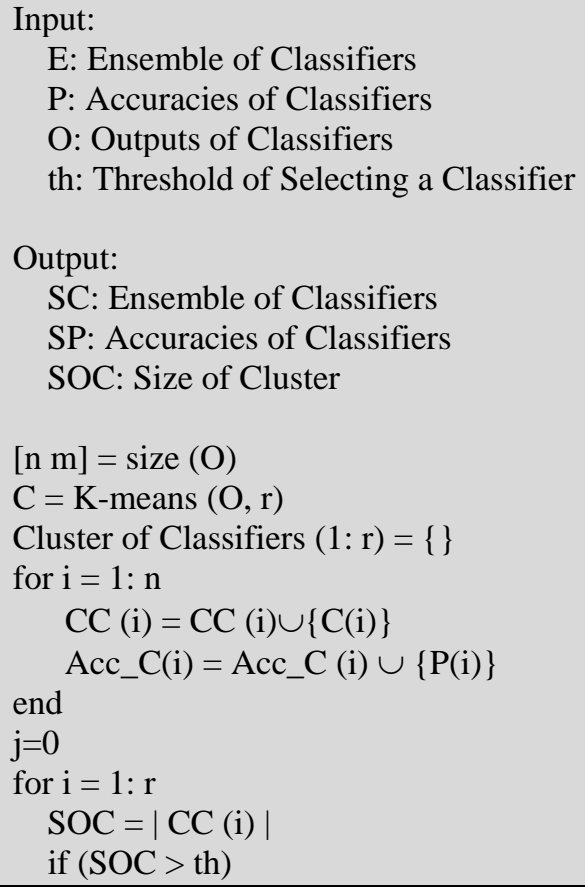

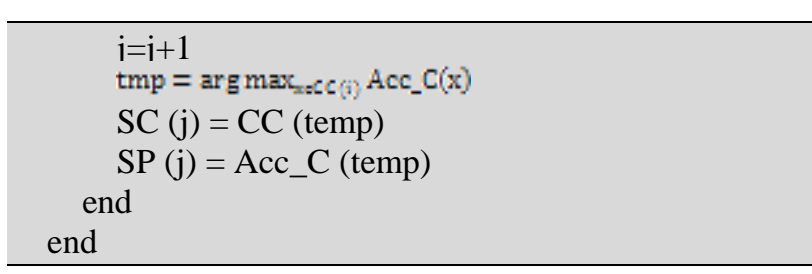

Fig. 5: Pseudo code of the proposed approach by selecting most accurate classifiers from each cluster

\subsection{Center-cluster Selection Mechanism}

The concept of classifiers clustering is reaching to more diversity. To provide more diversity, we proposed to choose the classifier which is the most closest to the center of each cluster. The Pseudo code of the proposed approach using this selection mechanism is shown in Fig. 6.

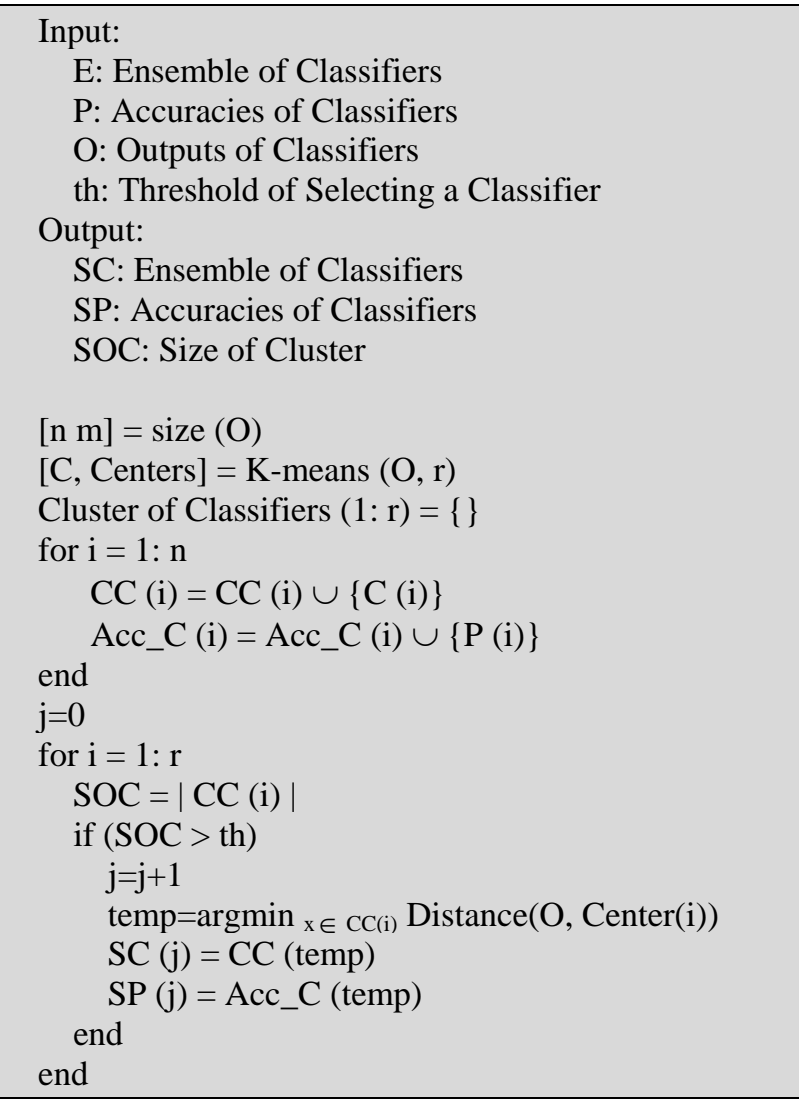

Fig. 6: Pseudo code of the proposed approach employing center-cluster selection mechanism

Until now, three methods for selecting classifier from each cluster have been described. To evaluate the performance of the proposed approach, we ran experiments on 18 representative datasets from the UCI repository. In this paper, the measure of performance employing different selecting methods is achieved for classifying the datasets. Furthermore, the effect of sampling rate and the number of clusters is investigated. 


\section{Experiments}

In this section, experimental results of employing our proposed approach using some standard datasets are reported. Accuracy of the classifiers is calculated by 4 fold-cross-validation method. A short explanation of datasets used in this research is given in Table 1.

Table 1: Short explanation of the examined datasets

\begin{tabular}{|c|c|c|c|}
\hline $\begin{array}{c}\text { Data } \\
\text { set }\end{array}$ & $\begin{array}{c}\text { Number of } \\
\text { classes }\end{array}$ & $\begin{array}{c}\text { Number of } \\
\text { features }\end{array}$ & $\begin{array}{c}\text { Number of } \\
\text { instances }\end{array}$ \\
\hline Iris & 3 & 4 & 150 \\
\hline Wine & 3 & 13 & 178 \\
\hline Yeast & 10 & 8 & 1484 \\
\hline SA-Heart & 2 & 9 & 462 \\
\hline Breast Cancer & 2 & 9 & 683 \\
\hline Bupa & 2 & 6 & 345 \\
\hline Glass & 6 & 9 & 214 \\
\hline Galaxy & 7 & 4 & 323 \\
\hline Half-Ring & 2 & 2 & 400 \\
\hline Ionosphere & 2 & 34 & 351 \\
\hline Balance Scale & 3 & 4 & 625 \\
\hline test Monk1 & 2 & 6 & 122 \\
\hline test Monk2 & 2 & 6 & 412 \\
\hline test Monk3 & 2 & 6 & 6 \\
\hline train Monk1 & 2 & 6 & 612 \\
\hline train Monk2 & 2 & 6 & 6 \\
\hline train Monk3 & 2 & 6 & 6 \\
\hline
\end{tabular}

All datasets are extracted from Machine Learning website [1] except for Halfring dataset which is handmade and is considered as a difficult dataset for machine learning algorithms [8]. All parameters of classifiers are constant during creation of an ensemble.

Decision tree (DT) is one of the most versatile classifiers in the Machine Learning field. Decision tree is considered as one of the unstable classifiers that can be suitable for ensemble construction. It uses a tree-like graph or model of decisions. The kind of representation is appropriate for experts to understand what classifier does [26]. Its intrinsic instability can be employed as a source of diversity in classifier ensemble. The ensemble of a number of DTs is a well-known algorithm called Random Forest (RF), which is one of the most powerful ensemble algorithms. The algorithm of Random Forest was first developed by Breiman [5]. In this paper, DT is totally used as base classifier. Criterion of decision tree is Gini criterion and parameter of pruning is set to 2 .
The Multi-Layer Perceptrons (MLP), the most representatives of artificial neural network, is a linear classifier for classifying data specified by parameters and an output function. Its parameters are adapted similar to stochastic steepest gradient descent. The units each perform a biased weighted sum of their inputs and pass this activation level through a transfer function to produce their output, and the units are arranged in a layered feed forward topology. The network thus has a simple interpretation as a form of input-output model, with the weights and thresholds (biases) the free parameters of the model. Important issues in MLP design include specification of the number of hidden layers and the number of units in these layers [24]. One way is to set the weights explicitly, using a prior knowledge. Another way is to 'train' the MLP, feeding it by teaching patterns and then letting it change its weights according to some learning rule. In this paper, the MLP is used as one of the base classifiers.

Number of hidden layers in neural network classifiers is set to 2 and the number of neurons in each layer is respectively 10 and 5 neurons. In each layer of the neural network, functions 'tansig', 'purelin' and 'logsig' is used.

Gianito [25] imply a clustering and selection method to deal with the diversity generation. In that work, at first, a large number of classifiers with different initializations are produced, and then a subset of them is selected according to their distances in their output space. This research doesn't take into consideration how the base classifiers are created.

In Table 2, the accuracy of the proposed approach using Bagging, Boosting and Gianito with sampling rate of $30 \%$, appropriate cluster threshold of 2 and basic classifiers of neural networks has been shown. The fifth column shows the Gianito method [25]. As shown from the sixth column (from left to right) with the proposed approach using selection of the most accurate classifiers from clusters and Bagging method, higher performance is achieved compared to the other methods. The eighth and ninth columns, respectively, shows the results of experiments using the proposed approach with the algorithm presented in Fig. 5 and Bagging and Boosting generators. The tenth and eleventh columns show the results of proposed approach using random selection of classifiers from each cluster and employing of Bagging and Boosting generators.

Table 3 shows the results of repeated experiments of Table 2 using decision trees as weak classifiers. What was concluded from Table 2 can be seen here too. As the results illustrated, the proposed approach with Bagging algorithm has great advantage over other ones. The degree of this advantage is higher here than what resulted from Table 2. This is for rather instability of basic classifiers of decision tree compared to artificial neural networks. The bold value in the row of each dataset shows the highest accuracy. 
Table 2: Accuracy of different classification (\%) with approaches of Bagging, Boosting and Gianito, sampling rate of 30\%, appropriate cluster threshold of 2 and basic classifiers are neural networks

\begin{tabular}{|c|c|c|c|c|c|c|c|c|c|c|}
\hline \multirow[b]{2}{*}{ Dataset } & \multirow[b]{2}{*}{$\begin{array}{c}\text { Ada } \\
\text { Boost }\end{array}$} & \multirow[b]{2}{*}{ Arc-X4 } & \multirow[b]{2}{*}{$\begin{array}{c}\text { Bagging } \\
\text { (Random } \\
\text { Forrest) }\end{array}$} & \multirow[b]{2}{*}{$\begin{array}{l}\text { Cluster } \\
\text { and } \\
\text { Selection } \\
\text { (Gianito) }\end{array}$} & \multicolumn{6}{|c|}{ Classifier Selection } \\
\hline & & & & & $\begin{array}{c}\text { Bagging } \\
\text { (Most } \\
\text { Accurate } \\
\text { Classifier } \\
\text { Selection) }\end{array}$ & $\begin{array}{c}\text { Arc-X4 } \\
\text { (Most } \\
\text { Accurate } \\
\text { Classifier } \\
\text { Selection) }\end{array}$ & $\begin{array}{l}\text { Bagging } \\
\text { (Center- } \\
\text { Cluster } \\
\text { Selection) }\end{array}$ & $\begin{array}{c}\text { Arc-X4 } \\
\text { (Center- } \\
\text { Cluster } \\
\text { Selection) }\end{array}$ & $\begin{array}{c}\text { Bagging } \\
\text { (Random } \\
\text { Classifier } \\
\text { Selection) }\end{array}$ & $\begin{array}{c}\text { Arc-X4 } \\
\text { (Random } \\
\text { Classifier } \\
\text { Selection) }\end{array}$ \\
\hline Iris & 94.67 & 96.62 & 96.62 & 93.33 & 98.66 & 97.32 & 97.32 & 95.32 & 97.97 & 97.33 \\
\hline Wine & 94.38 & 96.59 & 96.06 & 95.23 & 98.3 & 97.74 & 97.18 & 96.62 & 97.19 & 95.51 \\
\hline Yeast & 59.50 & 60.85 & 61.19 & 60.56 & 59.82 & 60.64 & 60.04 & 61.18 & 61.19 & 60.85 \\
\hline SA-Heart & 69.48 & 73.04 & 72.39 & 70.18 & 71.64 & 70.98 & 71.64 & 71.20 & 71.52 & 71.09 \\
\hline Breast Cancer & 96.49 & 97.06 & 96.91 & 96.19 & 97.36 & 97.36 & 97.06 & 96.92 & 96.91 & 96.47 \\
\hline Bupa & 68.41 & 70.06 & 71.22 & 71.98 & 66.08 & 68.40 & 67.82 & 67.82 & 72.09 & 68.02 \\
\hline Glass & 66.36 & 66.04 & 66.98 & 67.05 & 69.62 & 67.28 & 67.74 & 66.34 & 67.45 & 66.04 \\
\hline Galaxy & 85.14 & 87.00 & 85.62 & 87.00 & 85.74 & 86.68 & 85.12 & 85.74 & 85.62 & 84.52 \\
\hline Half-Ring & 97.25 & 97.25 & 95.75 & 97.25 & 97.24 & 97.25 & 97.24 & 97.24 & 97.25 & 97.25 \\
\hline Ionosphere & 89.17 & 90.03 & 88.51 & 88.51 & 95.14 & 94.3 & 95.72 & 96.28 & 90.31 & 87.64 \\
\hline Balance Scale & 93.12 & 93.27 & 91.99 & 95.75 & 93.92 & 92.32 & 92.16 & 94.24 & 91.35 & 92.95 \\
\hline Monk problem 1 & 98.99 & 98.06 & 92.23 & 98.34 & 100.00 & 98.78 & 98.54 & 98.04 & 98.43 & 97.87 \\
\hline Monk problem2 & 87.48 & 87.35 & 85.68 & 87.21 & 85.42 & 87.62 & 87.62 & 87.36 & 87.41 & 87.23 \\
\hline Monk problem3 & 96.84 & 97.09 & 95.87 & 96.77 & 99.26 & 98.78 & 96.84 & 97.32 & 97.33 & 96.99 \\
\hline Average & 85.52 & 86.45 & 85.50 & 86.10 & 87.02 & 86.90 & 86.58 & 86.56 & 86.57 & 85.70 \\
\hline
\end{tabular}

Table 3: Accuracy of different classification (\%) with approaches of Bagging, Boosting and Gianito, sampling rate of 30\%, appropriate cluster threshold of 2 and basic classifiers are decision trees.

\begin{tabular}{|c|c|c|c|c|c|c|c|c|c|c|}
\hline \multirow[b]{2}{*}{ Dataset } & \multirow[b]{2}{*}{$\begin{array}{c}\text { Ada } \\
\text { Boost }\end{array}$} & \multirow[b]{2}{*}{ Arc-X4 } & \multirow[b]{2}{*}{$\begin{array}{c}\text { Bagging } \\
\text { (Random } \\
\text { Forrest) }\end{array}$} & \multirow[b]{2}{*}{$\begin{array}{c}\text { Cluster } \\
\text { and } \\
\text { Selection }\end{array}$} & \multicolumn{6}{|c|}{ Classifier Selection } \\
\hline & & & & & $\begin{array}{l}\text { Bagging } \\
\text { (Most } \\
\text { Accurate } \\
\text { Classifier } \\
\text { Selection) }\end{array}$ & $\begin{array}{c}\text { Arc-X4 } \\
\text { (Most } \\
\text { Accurate } \\
\text { Classifier } \\
\text { Selection) }\end{array}$ & $\begin{array}{l}\text { Bagging } \\
\text { (Center } \\
\text { Cluster } \\
\text { Selection) }\end{array}$ & $\begin{array}{c}\text { Arc-X4 } \\
\text { (Center } \\
\text { Cluster } \\
\text { Selection) }\end{array}$ & $\begin{array}{c}\text { Bagging } \\
\text { (Random } \\
\text { Classifier } \\
\text { Selection) }\end{array}$ & $\begin{array}{c}\text { Arc-X4 } \\
\text { (Random } \\
\text { Classifier } \\
\text { Selection) }\end{array}$ \\
\hline Iris & 94.67 & 96.62 & 95.27 & 94.59 & 96.66 & 94.66 & 95.32 & 95.32 & 96.62 & 95.95 \\
\hline Wine & 96.63 & 96.07 & 97.19 & 92.61 & 97.18 & 93.82 & 97.18 & 97.74 & 97.19 & 95.51 \\
\hline Yeast & 54.78 & 53.17 & 53.98 & 54.51 & 55.32 & 55.98 & 54.78 & 53.22 & 53.98 & 52.09 \\
\hline SA-Heart & 67.32 & 70.00 & 71.30 & 68.04 & 73.58 & 71.2 & 72.06 & 67.52 & 72.61 & 69.70 \\
\hline Breast Cancer & 96.19 & 95.74 & 96.32 & 93.68 & 95.90 & 95.02 & 96.48 & 95.46 & 96.47 & 95.05 \\
\hline Bupa & 66.96 & 70.64 & 72.09 & 64.53 & 72.74 & 74.48 & 72.46 & 71.30 & 72.97 & 66.28 \\
\hline Glass & 70.09 & 65.04 & 70.28 & 60.85 & 71.48 & 65.42 & 70.08 & 70.08 & 70.28 & 62.26 \\
\hline Galaxy & 71.83 & 70.59 & 73.07 & 70.94 & 72.12 & 72.74 & 72.74 & 72.74 & 72.45 & 70.28 \\
\hline Half-Ring & 97.25 & 97.25 & 95.75 & 95.75 & 97.25 & 96.74 & 96.50 & 96.00 & 97.25 & 95.75 \\
\hline Ionosphere & 91.17 & 90.31 & 92.31 & 87.64 & 92.30 & 95.14 & 92.02 & 92.02 & 91.45 & 89.74 \\
\hline Balance Scale & 91.52 & 94.44 & 93.60 & 94.44 & 93.60 & 97.60 & 94.24 & 94.56 & 94.72 & 94.24 \\
\hline Monk problem1 & 98.03 & 98.11 & 97.49 & 98.34 & 97.08 & 98.04 & 98.54 & 98.78 & 98.76 & 97.37 \\
\hline Monk problem2 & 91.65 & 97.01 & 86.64 & 97.14 & 100.00 & 87.62 & 97.32 & 87.12 & 97.62 & 86.73 \\
\hline Monk problem3 & 95.51 & 87.29 & 96.92 & 87.31 & 99.26 & 92.22 & 96.84 & 87.12 & 96.97 & 96.34 \\
\hline Average & 84.54 & 84.45 & 85.16 & 82.88 & 86.71 & 85.17 & 86.27 & 84.21 & 86.38 & 83.38 \\
\hline
\end{tabular}

As expected, according to the experimental results, performance is improved by employing our proposed approaches. This was not unexpected because increasing the accuracy and diversity of the various classifiers, 
enhance the accuracy of ensembles. Besides using the most accurate classifiers form each cluster as representative, the proposed approach was led to greater efficiency. If we choose the best classifiers from each cluster, both criteria have been satisfied to make successful ensemble. Fig. 7 shows the effect of number of clusters and sampling rate on the performance of clustering algorithm using decision tree classifiers, Bagging generator on Iris, Bupa and Breast cancer datasets. The results illustrate that number of clusters greater than 11 lead to accuracy reduction. The reason is that the clusters are sparse and clustering them actually loses its meaning. As noted in this Fig. 7(b), the classification accuracy by increasing the sampling rate to $50 \%$ is encountered with increase, but after that, it reduced.

For example in the Iris dataset, up to the sampling rate of $50 \%$, the accuracy is increasing. This increment is not constant, therefore it can be concluded that from sampling rate $10 \%$ up to $50 \%$, accuracy uniformly and ascending is enhanced. Also with incrementing the number of clusters from 3 to 11, accuracy is increasing.

For Bupa dataset, up to the sampling rate of $50 \%$ the accuracy is increasing and this increment almost up to sampling rate of $70 \%$ continues but after that, the accuracy is reduced significantly. With increasing the number of clusters from 3 to 5, almost accuracy remained constant but then up to 11 , the accuracy increment is significant.

In the Breast cancer dataset, up to the sampling rate of $50 \%$, the accuracy is continuously increasing and thereafter accuracy increment is not permanent. With growing the number of clusters up to 9, accuracy is also increasing and it remained almost constant from 9 to 11 and then declined. Therefore, from the results it can be concluded that the best sampling rate is $50 \%$ and the best number of clusters is 11 . Number of clusters more than 11 , causes clusters to be scattered and decreases the accuracy.
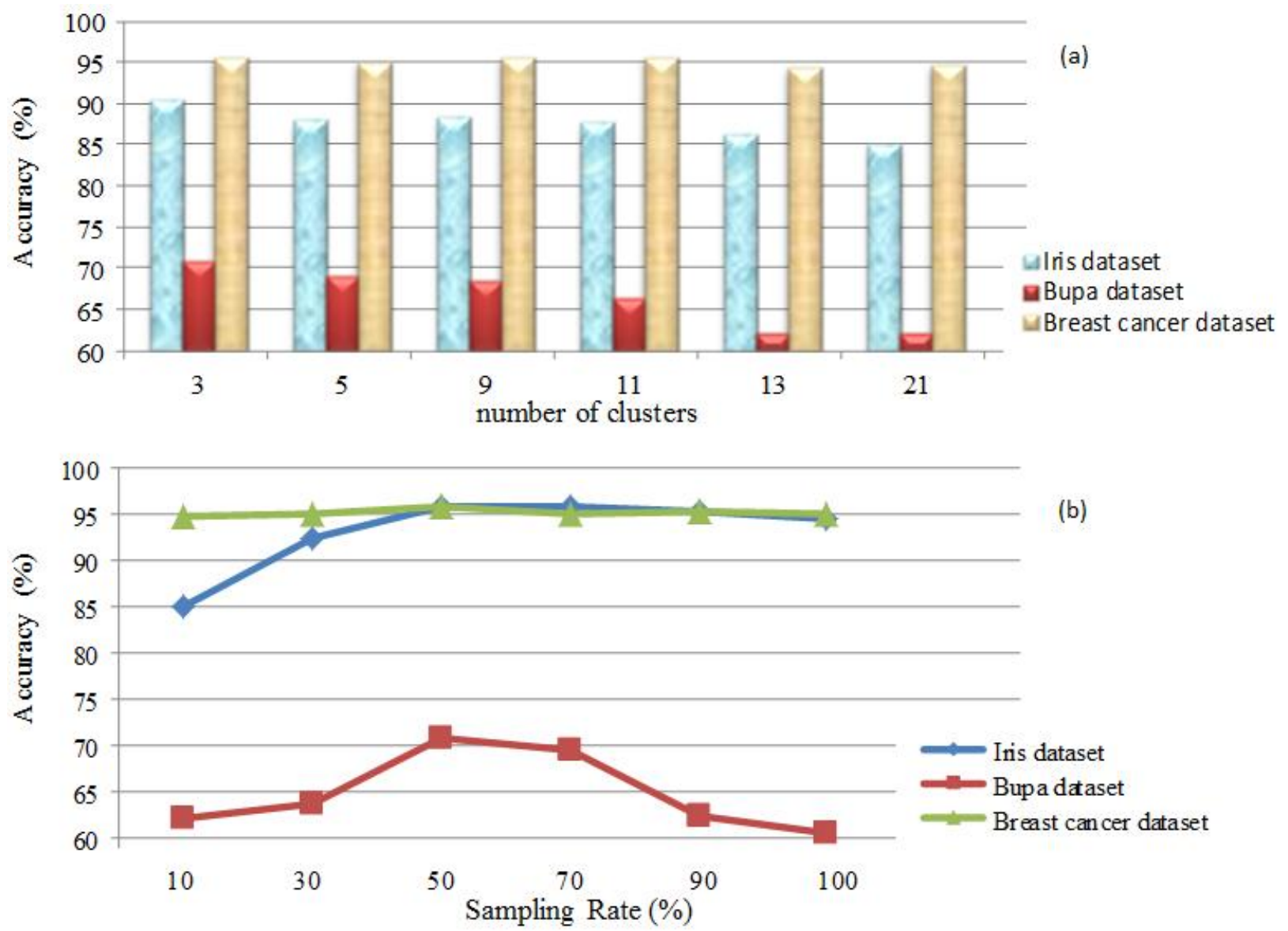

Fig. 7: (a) Effect of number of clusters (b) Effect of sampling rate on the performance of accurate selection method; Selection of most accurate classifiers from each cluster using decision tree as basis classifiers, Bagging generator and n=151(number of classifiers)

Fig. 8 shows comparison between average accuracy of Bagging and Boosting, considering effectiveness of the number of classifiers and sampling rate that respectively are setting to $(31,51,101,151)$ and $(10,30,50,70,90$, 100). As previously mentioned, using Bagging generator, performance of the proposed method is more highlights.
It is understood from Fig. 8 that employing Bagging generator in all selection methods results better than applying Boosting generator. Fig. 8 shows that the best sampling rate for both Bagging and Boosting generators is $50 \%$. 


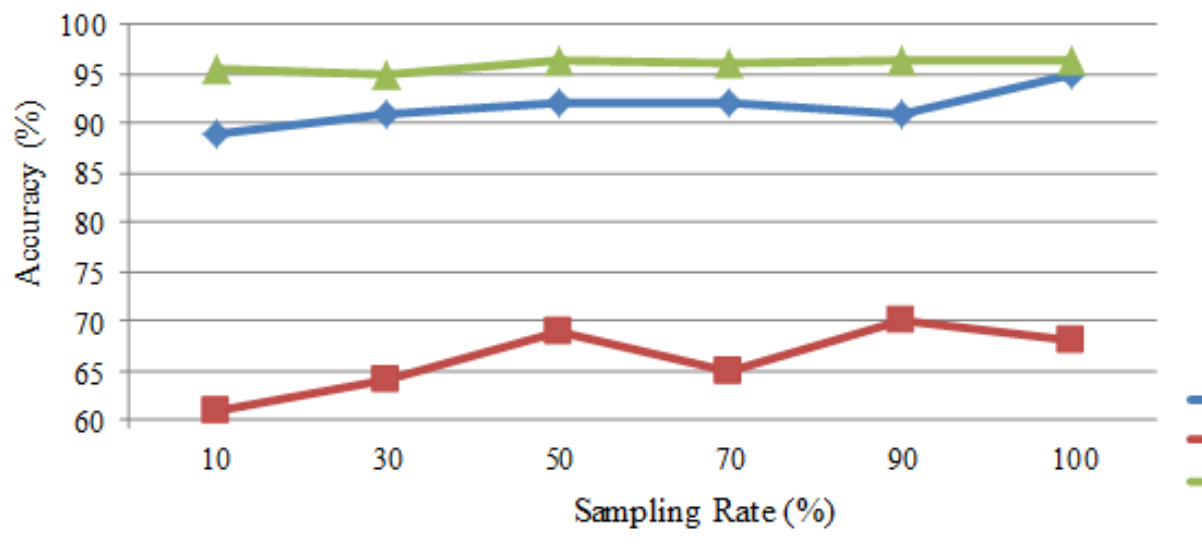

(a)

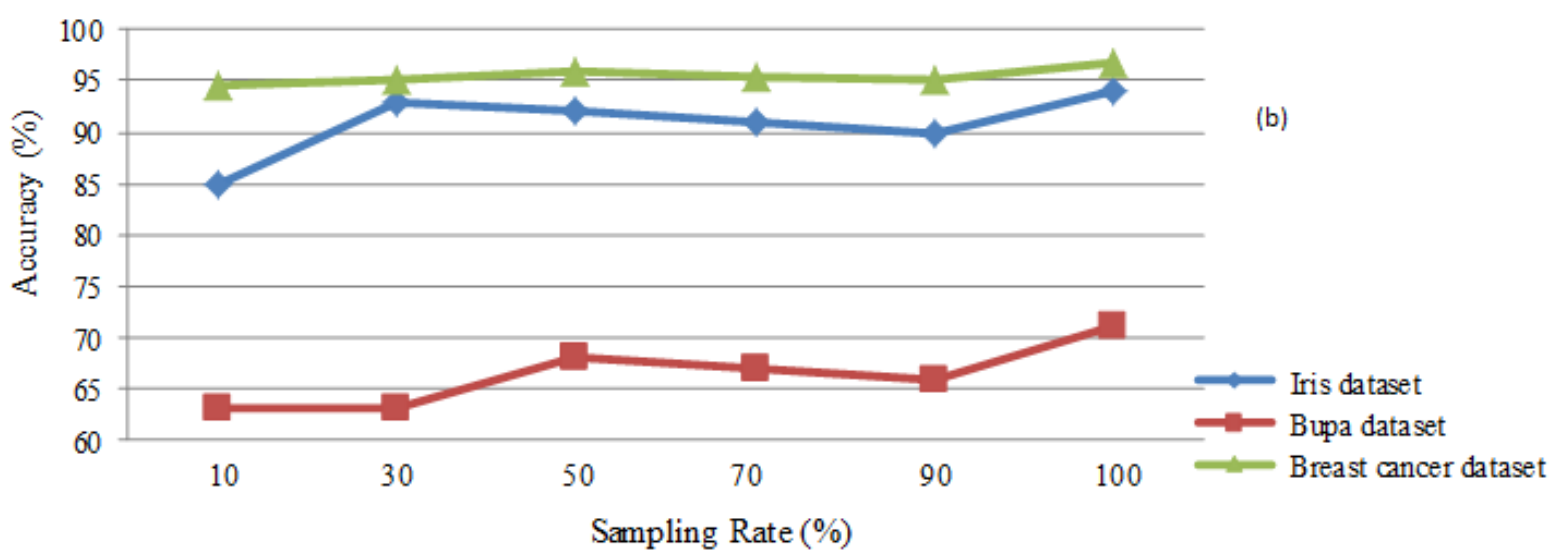

Fig. 8: Comparison of the average accuracy using Bagging and Boosting in all the selection methods with effectiveness of sampling rate by decision tree basis classifiers, (a) Average accuracy of Bagging on Iris, Bupa and Breast cancer datasets (b) Average accuracy of employing Boosting generator on Iris dataset, Bupa and Breast cancer datasets

\section{Conclusion}

In ensemble construction, all clustering and selection methods suffer from selecting an appropriate classifier from each cluster. In our proposed approach, the main goal is increasing the accuracy of classification. The accuracy of an ensemble could depend on sampling rate and number of clusters. In previous works, employing Bagging and Boosting methods, the value of sampling rate is always set to 100 . In our proposed approach, we investigated the effectiveness of different values of sampling rate on the accuracy of final constructed ensemble. Type of basic classifiers is decision tree or multi-layer neural networks. After constructing a large number of basic classifiers, our approach clusters them employing k-means clustering algorithm. Afterward, three selection methods are proposed for choosing proper classifiers from clusters. Furthermore, in this paper the effect of number of clusters on the performance of ensembles was investigated. The experiments demonstrate that sampling rates more than $50 \%$ not only do not increase classification accuracy but also decrease it. Moreover, the number of clusters greater than 11 lead to reduce accuracy. The reason is that the clusters get sparse and clustering them actually loses the meaning. Proposed approach with Bagging algorithm has great advantage over other ones. Experimental results show that picking the classifiers with highest accuracy from each cluster results the ensemble with the highest accuracy. Clustering ensures ensemble diversity and the best classifiers are selected from clusters, to have an accurate and varied ensemble.

\section{Reference}

[1] Roli F., Giacinto, "An Approach to the Automatic Design of Multiple Classifier Systems", Department of Electrical and Electronic Engineering, University of Cagliari, Italy, 2000.

[2] Thomas, G. Diettrech, "Ensemble method in machine learning", Proc. of First International Workshop on Multiple Classifier Systems, 2000, 115 .

[3] Hansen L., Salamon P., "Neural network ensembles", IEEE Transactions on Pattern Analysis and Machine Intelligence, 1990, 12: 993-1001.

[4] Khanchel R., Limam M., "Empirical comparison of Arcing algorithms", in proc. of Applied Stochastic Models and Data Analysis, 2000, 1433-1440. 
[5] Breiman L., "Bagging Predictors", Journal of Machine Learning, 1996, 24(2):123-140.

[6] Minaei-Bidgoli B., Topchy A.P., Punch W.F., "Ensembles of Partitions via Data Resampling", Proc. of Information Technology: Coding and Computing, 2004, 188-192.

[7] Minaei-Bidgoli B., beigi A., "A new classifier ensembles framework", Proc. of Knowledge-Based and Intelligent Information and Engineering Systems, Springer, 2011, 110-119.

[8] Minaei-Bidgoli B., William F. Punch, "Ensembles of Partitions via Data Resampling”, Proc. of International Conference on Information Technology: Coding and Computing, 2004.

[9] Simon Günter, Horst Bunke, "Creation of Classifier Ensembles for Handwritten Word Recognition Using Feature Selection Algorithms", Proc. of the 8th International Workshop on Frontiers in Handwriting Recognition, 2002.

[10] Tumer, K., Ghosh, J., "Error correlation and error reduction in ensemble classifiers", Connection Science, 1996, 8(4): 385-403.

[11] Kuncheva L., "Combining Pattern Classifiers, Methods and Algorithms", Wiley, 2005.

[12] Peña J.M., "Finding Consensus Bayesian Network Structures", Journal of Artificial Intelligence Research, 2011, 42: 661-687.

[13] Kuncheva, L.I. and Whitaker, C., "Measures of diversity in classifier ensembles and their relationship with ensemble accuracy", Journal of Machine Learning, 2003, 51(2): 181-207.

[14]Parvin H., Minaei-Bidgoli B., Shahpar H., "Classifier Selection by Clustering", Proc. of 3rd Mexican Conference on Pattern Recognition, LNCS, Springer, 2011, 60-66,.

[15] Yang T., "Computational Verb Decision tree", International Journal of Computational Cognition, 2006, 4(4): 34-46.

[16] Blake C.L., Merz C.J., "UCI Repository of machine learning databases", 1998.

[17] Breiman L., "Random Forests", Machine Learning, 2001, 45(1): 5-32.

[18] Giacinto, G., Roli, F., "An approach to the automatic design of multiple classifier systems", Pattern Recognition Letters, 2001, 22: 25-33.

[19] Sharkey, A.J.C., "On Combining Artificial Neural Nets", Connection Science, vol. 8, pp. 299-314, 1996.

[20] Amigó E., Gonzalo J., Artiles J., and Verdejo F., "Combining Evaluation Metrics via the Unanimous Improvement Ratio and its Application to Clustering Tasks", Journal of Artificial Intelligence Research, 2011, 42: 689-718.
[21] Freund Y., Schapire R.E., "A Decision-Theoretic Generalization of Online Learning and an Application to Boosting", Journal Computer Syst. Sci., 1997, 55(1): 119-139.

[22] Dietterich, T.G., "An experimental comparison of three methods for constructing ensembles of decision trees: Bagging, Boosting, and randomization", Machine Learning, 2000, 40(2): 139-157.

[23] Dasgupta S., "Which Clustering Do You Want? Inducing Your Ideal Clustering with Minimal Feedback", Journal of Artificial Intelligence Research, 2010, 39: 581-632.

[24] Haykin S., "Neural Networks, a comprehensive foundation", 1999.

[25] Gunter S., Bunke H., "Creation of classifier ensembles for handwritten word recognition using features selection algorithms", 2002.

\section{Authors' Profiles}

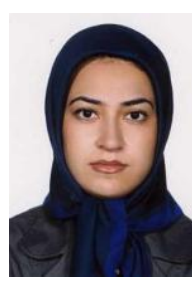

Hedieh Sajedi: Dr. Hedieh Sajedi received a B.Sc. degree in Computer Engineering from AmirKabir University of Technology in 2003, and M.Sc. and Ph.D degrees in Computer Engineering (Artificial Intelligence) from Sharif University of Technology, Tehran, Iran in 2006 and 2010, respectively. She is currently an Assistant Professor at the Department of Computer Science, University of Tehran, Iran. Her research interests include Pattern Recognition, Machine Learning and Signal Processing.

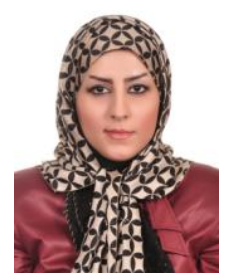

Elham Masoumi: She received M.Sc. in Computer Engineering from Department of Computer Engineering and Information Technology, Qazvin Branch, Islamic Azad University of Iran. She currently has lectures in computer science at University of Applied Sciences. Her current research interests include distributed data mining and classifiers combination. 